\title{
Cloud Detection of Gray Photographs by Cloud Region Detectionand Optimal Region Grow Threshold
}

\author{
Xingying Zhao ${ }^{\mathrm{a}}$, Hong Zheng ${ }^{\mathrm{b}}$ \\ School of Automation Science and Electrical Engineering, Beihang University, Beijing, China \\ a zhaoxingying123@126.com, bjulyanna@vip.sina.com
}

\begin{abstract}
The most cloud detection method depends on various bands such as thermal band. In this paper, we propose an automatic cloud detection algorithm for gray photographs. Based on the properties of cloud derived from gray photographs and the human visual perception, we present a novel scheme for detecting clouds in the gray photographs. We first cut an input graph into many pieces. Through the conditional contrast and area feature, we label these pieces into thick cloud, thin cloud and no cloud. We select the point of maximal gray as seed point and calculate the optimal threshold. Then, we consider the texture feature of the growing area and fix some errors of detection results. Finally, we get the detected result. The algorithm is evaluated in terms of aerial photograph scenes with manually generated cloud masks was created, and the evaluation results show that the method works well for the cloud detection of gray photographs.
\end{abstract}

Keywords-Cloud detection; Gray photograph; Human visual perception; Conditional contrast; Region grow; Optimal threshold; Texture

\section{INTRODUCTION}

The satellite remote sensing images are a resource for global change research and applications in agriculture, cartography, geology, forestry, regional planning and surveillance. The presence of clouds affects the use of satellite data and leads to inaccurate analysis for the images. So, cloud detection is an important preprocessing for many follow-up manipulations, such as object recognition, image retrieval, and image classification.

There are several challenges facing cloud detection for gray image. First and foremost is the limited information and complexity of images. It is essentially an image segmentation problem and the only information is the intensity of pixels. However, for complex aerial images there are both bright no cloud regions and thin cloud pixels. Because of the limited image information and complex situation, it is difficult to get a satisfactory segment result.

The second challenge is the determination of cloud boundary. Even if we get the accurate estimated result of all pieces and give the right seed points, we may not obtain the ideal growing boundary of cloud. For local consideration, the growth threshold of each cloud may be different. As a result, we need to automatically select the optimal growth threshold according to different situations.

The third challenge for cloud detection of gray image is that most proposed methods are designed for moderate spatial resolution sensors. These sensors can collect data of different wavelengths. So, most of the work is focus on the cloud detection of multi-spectral images and little work on the cloud detection of gray image with just gray band.

The scope of this paper, therefore, is to describe the development of an algorithm for automatic cloud detection, using the gray level image while creating a cloud mask, with only a few minutes of run time. There are three main contributions in this paper:

1) We develop a cloud detection method to distinguish each $64 * 64$ piece to a certain class, including thick cloud, thin cloud and surface feature.

2) We propose an optimal threshold setting scheme to obtain a good boundary of cloud detection

3) We introduce a method to find the wrong regions and correct it, which can reduce the part of the object being judged as a cloud.

\section{RELATED WORK}

In the most research, many automatic cloud detection methods are explored for multi-spectral satellite images. They are highly dependent on the available spectral bands. Some of these methods work on a pixel-by-pixel basis [4], and some use neighborhood information, such as local standard deviation [1]. The ACCA algorithm is used for cloud assessment of TM and ETM+ data. It was developed by the Landsat Project Science Office at NASA's Goddard Space Flight Center (GSFC). There are 8 bands of different wavelength, with a spatial resolution between $15 \mathrm{~m}$ and 100 $\mathrm{m}$. Based on the Landsat data, many cloud detection methods [2], [3] have been proposed to improve the detection performance.

\section{Cloud Detection Algorithm}

In gray photographs, human is easy to identify clouds from others. However, identifying clouds by computer is a difficult job, particularly detecting the cloud regions automatically. After a large amount of observations and statistical experiment we have observed that the cloud pieces in the gray image usually have the following common properties:

- Property 1: Cloud regions generally have higher intensity since the reflectivity of cloud regions is usually larger than that of the non-cloud regions.

- Property 2: Human often identify cloud regions via local contrast, so for the computer, local contrast is an important property to identify cloud with lower intensity.

- Property 3: The texture of cloud regions is often different with ground object features, and the edge of a piece image with cloud region is irregular. 
Based on the aforementioned properties, we propose our for cloud detection. In Fig. 1, we give the block diagram of the proposed cloud detection system.

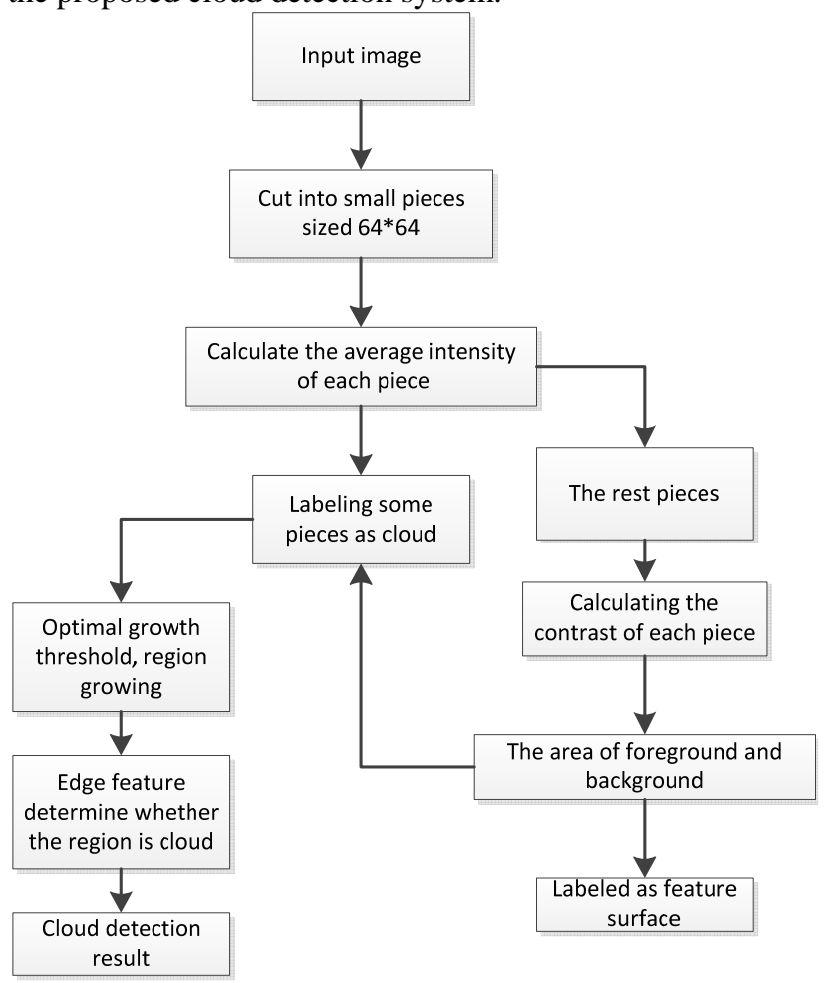

Figure 1. Block diagram of the proposed cloud detection algorithm

Our system has the following main steps. First, we cut an input image, usually sized $1024 * 1024$, into several $64 * 64$ pieces, and label the pieces with very high intensity average as category one, which means the whole piece is a high intensity cloud region. Second, intensity can't distinguish thin cloud pieces from the image, so we use contrast and area as main feature to label the pieces as surface feature and cloud. Furthermore, all pieces that are decided as cloud region can get a seed point, and calculate an optimal threshold as growing threshold for the input image. Finally, we consider the texture of the growing area and fix some errors in the results.

\section{A. Average Intensity}

According to the aforementioned observations (property 1 ), the cloud pixels usually have comparatively high intensities. So for a piece of image that is full of thick cloud, the average intensity is higher than others. To identify the distribution law of intensity true cloud pieces, we collect 30327 pieces of cloud region and 7029 pieces of no cloud region from different images. After calculating the average intensity of each piece, we plot the histogram of intensity based on the average value of all pieces of two categories. Figure 2(a) is the intensity histogram over all cloud pieces. We can see that over $15 \%$ of the cloud pieces have intensity not less than 200 and there are no cloud pieces with average intensity less than 40. Figure 2(b) is the histogram of non-cloud pieces.

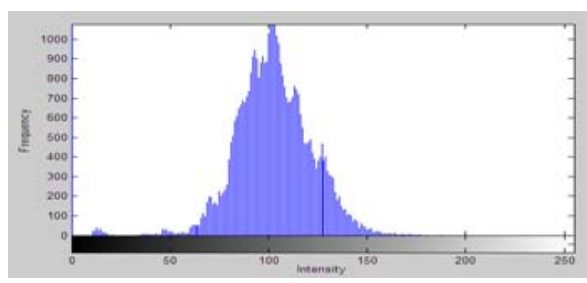

(a)

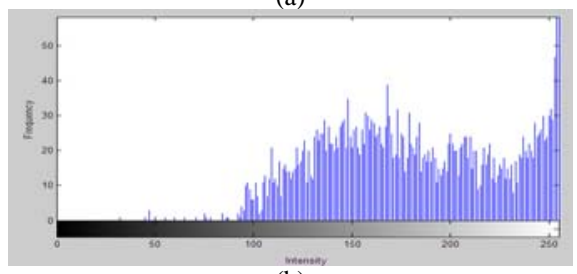

(b)

Figure 2. The average intensity of small pieces

Notice that, to decrease the complex situation of cloud and surface feature pieces, we first label some pieces by average intensity that is indeed cloud pieces and feature pieces. As can be seen from the statistical results, if a pieces with average intensity more than 200, it is a piece of thick cloud. On the other hand, if the average intensity of a piece is less than 40 , it is a piece of feature surface.

\section{B. Contrast Calculate}

Intensity can only distinguish thick cloud pieces from others. So, for the bright non-cloud region and thin cloud region, we propose a new method that is calculate the contrast of each piece.

Contrast is the local change in brightness and is defined as the ratio between average brightness of an object and the background. Strictly speaking, we should talk about luminance instead of brightness if our aim is to be physically precise. The human eye is logarithmically sensitive to brightness, implying that for the same perception, higher brightness requires higher contrast. Apparent brightness depends very much on the brightness of the local surroundings this effect is called conditional contrast [5].

For a piece of image, the human eye judging whether there is a cloud region by contrast partly. In order to calculate the contrast correctly, the foreground and background are needed, and the average gray of foreground and background is calculated respectively. The result of contrast is the ratio of average gray of foreground and background. Here, we define the foreground as cloud pixels, background for the ground surface pixels. So we need to know about the foreground region and the background region in the small pieces. Considering the gray value of cloud pixels is always higher than the feature, we select the point with maximal intensity as the seed point for 8 neighborhood region growing. Because we don't need very precise result, we roughly set the growth threshold as maximal intensity minus 25. 
First, the pieces with cloud region always grow out of two regions. Because the thick cloud pieces with symmetrical intensity are considered at average intensity step. So, in this step, if an image piece grows into one region, it can be considered as feature.

Second, there are some bright non-cloud regions indeed, and the contrasts of them are relatively high, but the areas of them are smaller than the cloud regions.

In this step, we don't need accurate determination of each piece. The first reason is that subsequent steps can correct the wrong result. The second is because we don't need to find all cloud pieces. Every cloud, as long as there is a mark, it can be grown from seed point in a later step completely. So we determine the threshold of contrast, area and average Intensity based on statistical results.

Based on the statistical result, we design a pass to label cloud pieces and feature ground pieces, the process showing in Figure 3.

If the contrast equal to zero, it indicate that the piece is a feature piece with well-distributed intensity because we labeled the well-distributed cloud pieces in step A. If the contrast is more than 1.22, the area of foreground is more than 512 and the foregound average intensity is more than 100 , we label it as cloud region.

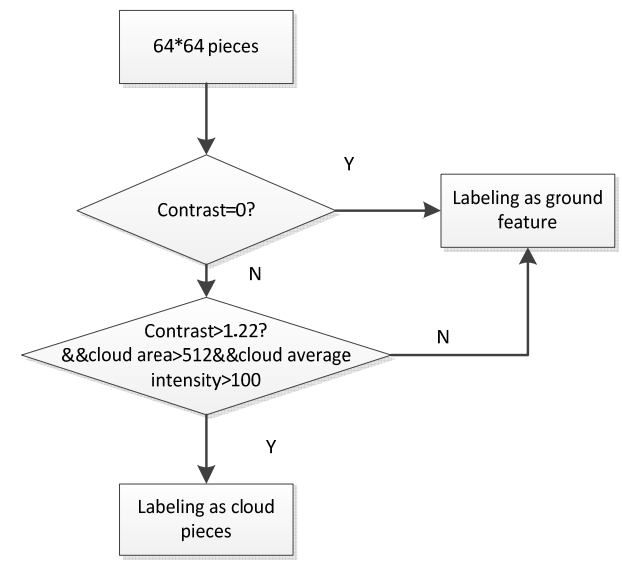

Figure 3. Labeling process by contrast and area

\section{Region Grow and Optimal Threshold}

The steps mentioned before distinguish cloud pieces from others, and choose the point with highest intensity as seed point. In this part, we define an energy function to calculate the optimal growth and get the optimal growth boundary.

For the thick cloud pieces in step one, we set the growth threshold 45, and obtain the growth result.

For the thin cloud pieces, growth threshold range from 10 to 65 and the step size is 5 . Assuming the threshold is $\mathrm{H}$, the intensity value of the seed points is MaxGray. Getting a value GrayLevel $=$ MaxGray $-\mathrm{H}$.

Taking the seed point as the starting point, and grow as the 8 neighborhood. So long as the intensity value of the pixel is higher than or equal to GrayLevel, the pixel is considered as cloud pixel. Then we get two regions, the cloud region and the feature region. Along the edge, in the gradient direction of the edge, we take three pixels from cloud region and three pixels from feature region respectively. For digital images, gradient magnitude and direction is:

$$
\begin{gathered}
G[f(i, j)]=\left[\left(\Delta_{x} f(i, j)^{2}+\left(\Delta_{y} f(i, j)^{2}\right]^{1 / 2}\right.\right. \\
\alpha=\arctan \left[\Delta_{y} f(i, j) / \Delta_{x} f(i, j)\right]
\end{gathered}
$$

Where,

$$
\begin{aligned}
& \Delta_{x} f(i, j)=f(i, j)-f(i-1, j) \\
& \Delta_{y} f(i, j)=f(i, j)-f(i, j-1)
\end{aligned}
$$

Then the energy function is defined as:

$$
\begin{aligned}
& C\left(\varphi, a_{1}, a_{2}\right)=\int\left(I_{C}(x, y)-a_{1}\right)^{2} d x d y+ \\
& \int\left(I f(x, y)-a_{2}\right)^{2} d x d y
\end{aligned}
$$

$I_{c}(x, y)$ represents the intensity of the interior pixels of gradient direction of the edge, $I_{f}(x, y)$ is the intensity of the exterior pixels and $a_{1}$ and $a_{2}$ represent the mean intensities of the interior and exterior pixels of the edge. When the result of the function changes slowly, we think that this is the most appropriate edge. When the energy value changes slowly, it indicates edge coincides with the object boundary and best separates the object and background because the threshold increment doesn't have effect on the energy function value with respect to their mean intensities near edge. In this case the corresponding threshold is the optimal growing threshold.

\section{Texture Feature}

Because the cloud shields the feature, it will result in discontinuous texture feature. And quantifying the texture content of a region is an important approach for describing this region. So, in this step, we consider the texture of the region has detected by the steps mentioned previously.

An approach used frequently for texture analysis is based on statistical properties of the intensity histogram. One class of such measures is based on statistical moments of intensity values. The expression for the nth moment about the mean is given by

$$
\mu_{n}=\sum_{i=0}^{L-1}\left(z_{i}-m\right)^{n} p\left(z_{i}\right)
$$

Where $Z_{i}$ is a discrete random variable indicating intensity levels in a region, $P\left(Z_{i}\right)$ is the corresponding normalized histogram, and $\mathrm{L}$ is the number of possible intensity value.

And $\mathrm{n}$ is the moment order, and $\mathrm{m}$ is the mean: 


$$
m=\sum_{i=0}^{L-1} z_{i} p\left(z_{i}\right)
$$

Then, we compute the texture measures in TABLE I.

TABLE I. DESCRIPTORS OF TEXTURE BASED ON INTENSITY HISTOGRAMS

\begin{tabular}{|l|l|l|}
\hline Moment & Expression & \multicolumn{1}{|c|}{ Measure of Texture } \\
\hline Standard deviation & $\sigma=\sqrt{\mu_{2}}=\sqrt{\sigma^{2}}$ & A measure of average contrast \\
\hline Third moment & $\mu_{3}=\sum_{i=0}^{L-1}\left(z_{i}-m\right)^{3} p\left(z_{i}\right)$ & $\begin{array}{l}\text { Measures the skewness of a histogram. This measure is } \\
0 \text { for symmetric histograms; positive by histogram } \\
\text { skewed to the right about the mean; and negative for } \\
\text { histograms skewed to the left. }\end{array}$ \\
\hline Uniformity & $U=\sum_{i=0}^{L-1} p^{2}\left(z_{i}\right)$ & $\begin{array}{l}\text { Measures uniformity. This measure is maximum when } \\
\text { all intensity values are equal (maximally uniform) and } \\
\text { decreases from there. }\end{array}$ \\
\hline Entropy & $\ell=-\sum_{i=0}^{L-1} p\left(z_{i}\right) \log _{2} p\left(z_{i}\right)$ & A measure of randomness \\
\hline
\end{tabular}

Along the boundary of the detected region, in the gradient direction of the region boundary, we take three pixels from detected region and three pixels from region look as feature surface region respectively. The pixels inside the boundary consist of set 1 , and the pixels outside the boundary consist of set 2 , and set 3 is the union of the pixels in set 1 and set 2 . According to the above described, we calculate the standard deviation, third moment, uniformity and entropy of the three set. For each region detected, we obtain 12 values.

We find 50 cloud regions and 50 feature surface regions as training samples and choose neural network as the classifier.

The 12 values mentioned previously is input of neural network, the output is two categories, that is cloud region and feature region. And there are 6 nodes in the hidden layer. Using the method in [6], we get a simple artificial neural network, when there is a new input, we can get the category of it. If the output is cloud region, we keep the detection result. If the output is feature region, we correct the region by input image.

\section{RESUlT AND DisCUSSION}

In this section, we further demonstrate the effectiveness of our cloud detection algorithm by both visual comparisons and quantitative evaluation.

We compare our method with the cloud detection method ACCA [7]. Furthermore, using the manual mask, we present a quantitative evaluation of the detection accuracy. In Figure 4, we compare our method with [7], which automatically detects cloud based on mutil-band. We observe that out method can produce satisfactory results for complex gray photographs especially for the thin cloud. To quantitatively evaluate the efficiency of our cloud detection method, we use the error rate to evaluate the accuracy of our method. The true positive rate is defined as:

$$
T P R=\frac{T P}{T P+F N}
$$

Where TP denotes the number of true positive pixels, it is say the cloud pixels are detected as cloud. And FN denotes the number of false negative pixels, which means that the cloud pixels are detected as feature.

The false positive rate is defined as:

$$
F P R=\frac{F P}{F P+T N}
$$

Where FP denotes the number of false positive pixels, which means that the feature pixels is detected as cloud.

And the true rate is defined as:

$$
A C C=\frac{T P+T N}{T P+F N+F P+T N}
$$

Where $\mathrm{TN}$ is true negative, which means the feature pixels are detected as feature.

The Table II shows the TPR, FPR and ACC of our method. It denotes that this method has a low error rate. And the method has a good performance on distinguishing a lot of surface feature pixels (usually have high intensities) from cloud pixels and they are also strong in detecting the semitransparent cloud pixels.

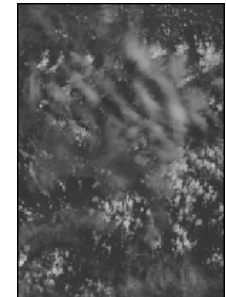

(a)

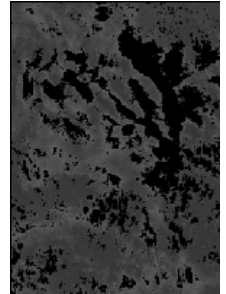

(b)

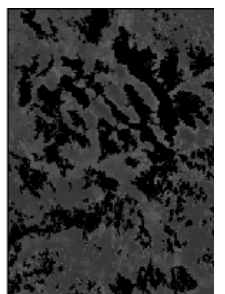

(c)
Figure 4. Comparisons with another competing cloud detection method [7]. (a) Input image. (b) Result of [7]. (c) Our result. 
TABLE II.

THE DETECTED RESULT OF OUR ALGORITHM

\begin{tabular}{lll}
\hline TPR & FPR & ACC \\
\hline $\mathbf{9 5 . 4 \%}$ & $0.2 \%$ & $96.6 \%$ \\
\hline
\end{tabular}

\section{CONCLUSION}

The results given here have shown that the cloud-cover algorithm we proposed is possible, only using the intensity of the image, with a good performance.

We first obtained the data set from internet. Then we create four steps for this data set and quantify the performance of our algorithm which correctly classifying $95.4 \%$ of the 500 aerial photographs, including $1.05 \times 10^{5}$ pixels validation set.

We cut the image into the number of pieces, and distinguish some pieces by intensity at first. However, the intensity just classifies a small part of pixel. So we calculate the contrast and area feature of the rest pieces and divide them into two parts: feature or cloud. After these steps, we classify all pieces into cloud and feature pieces. The correctly of classifying is $80.7 \%$.

Using the result of the pieces, we obtain a seed point of each piece, that is the brightest point of the piece. We define an energy function to optimize the growth threshold to get the most appropriate boundary of cloud.

Finally, according to the texture continuous of growth region and the outside region, we correct some error that the feature is detected as cloud. That is the result of our cloud detection algorithm.
In the future, a method that can distinguish ice from cloud is necessary. The texture of image is still an important feature.

\section{ACKNOWLEDGMENT}

The authors would like to thank Prof. Zheng for providing high-quality aerial photographs and her feedback and advice.

\section{REFERENCES}

[1] R. Saunders and K. Kriebel, "An improved method for detecting clear sky and cloudy radiances from AVHRR data,” Int. J. Remote Sens., vol. 9, no. 1, pp. 123-150, 1988.

[2] R. A. Frey, S. A. Ackerman, Y. Liu, K. I. Strabala, H. Zhang, J. R. Key, and X. Wang, "Cloud detection with MODIS. Part I: Improvements in the MODIS cloud mask for collection 5," J. Atmosp. Ocean. Technol., vol. 25, no. 7, pp. 1057-1072, Jul. 2008.

[3] S. Ackerman, R. Holz, R. Frey, E. Eloranta, B. Maddux, and M. McGill, "Cloud detection with MODIS. Part II: Validation," J. Atmosp. Ocean. Technol., vol. 25, no. 7, pp. 1073-1086, 2008.

[4] Y. Y. Boykov and M.-P. Jolly, "Interactive graph cuts for optimal boundary \& region segmentation of objects in nd images,” in Proc. 18th IEEE ICCV, 2001, vol. 1, pp. 105-112.

[5] Milan Sonka, Vaclav Hlavac, Roger Boyle, Image Processing, Analysis, and Machine Vision,3rd ed, vol.1. CL Engineering, 2007, pp.25-26.

[6] Bishop C.M. Pattern Recognition and Machine, vol.1. New York:Verlag New York Inc, 2006, pp.242-247.

[7] B. Hollingsworth, L. Chen, S. Reichenbach, and R. Irish, "Automated cloud cover assessment for Landsat TM images,” in Proc. SPIE Conf., vol. 2819, Imaging Spectrometry II , 1996, pp. 170-179. 